\title{
Ventricular strain analysis in patients with no structural heart disease using a vendor- independent speckle-tracking software
}

Hongmei Xia ${ }^{1}$, Darwin F. Yeung ${ }^{2}$, Cristina Di Stefano ${ }^{3}$, Stephen S. Cha ${ }^{4}$, Patricia A. Pellikka², Zi Ye², Jeremy J. Thaden ${ }^{2}$ and Hector R. Villarraga ${ }^{2^{*}}$ (D)

\begin{abstract}
Background: Ventricular strain measurements vary depending on cardiac chamber (left ventricle [LV] or right ventricle [RV]), type of strain (longitudinal, circumferential, or radial), ventricular level (basal, mid, or apical), myocardial layer (endocardial or epicardial), and software used for analysis, among other demographic factors such as age and gender. Here, we present an analysis of ventricular strain taking all of these variables into account in a cohort of patients with no structural heart disease using a vendor-independent speckle-tracking software.

Methods: LV and RV full-thickness strain parameters were retrospectively measured in 102 patients (mean age $39 \pm$ 15 years; $62 \%$ female). Within this cohort, we performed further layer-specific strain analysis in 20 subjects. Data were analyzed for global and segmental systolic strain, systolic strain rate, early diastolic strain rate, and their respective time-to-peak values.

Results: Mean LV global longitudinal, circumferential, and radial strain values for the entire cohort were $18.4 \pm 2.0 \%,-22.1 \pm 4.1 \%$, and $43.9 \pm 12.1 \%$ respectively, while mean RV global and free wall longitudinal strain values were $-24.2 \pm 3.9 \%$ and $-26.1 \pm 5.2 \%$ respectively. Women on average demonstrated higher longitudinal and circumferential strain and strain rate than men, and longer corresponding time-to-peak values. Longitudinal strain measurements were highest at the apex compared with the mid ventricle and base, and in the endocardium compared with the epicardium. Longitudinal strain was the most reproducible measure, followed closely by circumferential strain, while radial strain showed suboptimal reproducibility.
\end{abstract}

Conclusions: We present an analysis of ventricular strain in patients with no structural heart disease using a vendor-independent speckle-tracking software.

Keywords: 2D speckle-tracking echocardiography, Strain, Myocardial mechanics

\footnotetext{
* Correspondence: villarraga.hector@mayo.edu

${ }^{2}$ Department of Cardiovascular Diseases, Mayo Clinic, Rochester, Minnesota, 200 First St SW, Rochester, MN 55905, USA

Full list of author information is available at the end of the article
}

(c) The Author(s). 2020 Open Access This article is licensed under a Creative Commons Attribution 4.0 International License, which permits use, sharing, adaptation, distribution and reproduction in any medium or format, as long as you give appropriate credit to the original author(s) and the source, provide a link to the Creative Commons licence, and indicate if changes were made. The images or other third party material in this article are included in the article's Creative Commons licence, unless indicated otherwise in a credit line to the material. If material is not included in the article's Creative Commons licence and your intended use is not permitted by statutory regulation or exceeds the permitted use, you will need to obtain permission directly from the copyright holder. To view a copy of this licence, visit http://creativecommons.org/licenses/by/4.0/ The Creative Commons Public Domain Dedication waiver (http://creativecommons.org/publicdomain/zero/1.0/) applies to the data made available in this article, unless otherwise stated in a credit line to the data. 


\section{Background}

Strain and strain rate are sensitive measures of myocardial function that allow early detection of systolic dysfunction even before changes in ejection fraction (EF) [1-6]. However, strain measurements vary depending on cardiac chamber (left ventricle [LV] or right ventricle [RV]), type of strain (longitudinal, circumferential, or radial), ventricular level (basal, mid, or apical), myocardial layer (endocardial or epicardial), software used for analysis, and demographic factors such as age and gender [7-18].

Previous studies have reported reference values for strain, taking some but not all of these parameters into account $[7,10-13,16-21]$. Furthermore, many of these studies were performed in healthy subjects and therefore do not include the population of patients we encounter in practice who are referred for echocardiography for a clinical indication.

Here, we present an analysis of ventricular strain using a vendor-independent speckle-tracking software in a single cohort of patients who were referred for echocardiography but were found to have no overt evidence of structural heart disease.

\section{Methods}

\section{Study population}

We retrospectively screened 106 patients who were identified as having "normal echocardiograms" in our institutional echocardiography database between April 2009 and January 2014. The echocardiograms screened therefore represented a convenience sample of studies in which the interpreting cardiologist deliberately labeled the study "normal" to draw attention to the lack of any identifiable abnormality found using conventional echocardiographic parameters. These patients would therefore demonstrate the following based on the American Society of Echocardiography guidelines: normal ventricular size, wall thickness, and function including normal LV diastolic function; absence of ventricular wall motion abnormalities; estimated right ventricular systolic pressure within normal limits; no evidence of valvular stenosis; no more than physiologic regurgitation; normal-sized great vessels; no evidence of constriction; no more than physiologic pericardial effusion; and no evidence of congenital heart disease [22-26]. Exclusion criteria included a history of cardiovascular disease, evidence of cardiomyopathy on echocardiogram, non-sinus rhythm, or suboptimal 2D imaging quality.

Patients were referred for echocardiography for a variety of clinical indications, which fall under three broad categories: Group 1 patients were those who were referred for cardiac symptoms (chest pain, dyspnea, palpitations, or syncope), an abnormal electrocardiogram, or for a baseline evaluation prior to chemotherapy initiation;
Group 2 patients were those who had a family history of cardiomyopathy including hypertrophic cardiomyopathy, cardiac amyloidosis, idiopathic dilated cardiomyopathy, or noncompaction cardiomyopathy; and Group 3 patients were those who had systemic conditions or prior exposures in which screening for cardiomyopathy is recommended including systemic amyloidosis, hypereosinophilia, muscular dystrophy or suspected mitochondrial disorders, or prior chemotherapy or radiation therapy (Supplementary Table 1).

\section{Image acquisition and strain analysis}

All patients underwent a transthoracic echocardiographic examination with a standard commercially available ultrasound system [Vivid 7, General Electric (GE) Medical Systems, Fairfield, Connecticut] and a 1.5-4.3 $\mathrm{MHz}$ M4S transducer. Mean frame rates were 54.4 \pm $10.9 \mathrm{~Hz}$ for grayscale imaging. Strain measurements were performed offline with the vendor-independent 2D Cardiac Performance Analysis speckle-tracking software (Image Arena version 4.6, TomTec Imaging Systems, Unterschleissheim, Germany) from archived studies in Digital Imaging and Communications in Medicine (DICOM) format (Figs. 1 and 2).

Full-thickness strain measurements were performed for all patients in the cohort. The points in the cardiac cycle in which the ventricles reached maximum and minimum volumes represented end-diastole and endsystole respectively. LV longitudinal strain parameters were measured from the apical 4-chamber, 2-chamber, and 3-chamber views (Fig. 1). Circumferential and radial strain parameters were measured from the parasternal short-axis view at the mid-papillary level (Figure 2) as described in previous studies [12, 16, 17]. The LV was divided into six segments in each view. Full-thickness LV global longitudinal, circumferential, and radial systolic strain measurements were further compared between men and women, and between pre-defined age categories $(<40,40-59$, or $\geq 60$ years of age).

RV longitudinal strain parameters were measured from the modified RV-focused apical 4-chamber view. Global RV longitudinal strain was evaluated by averaging peak strain values of six segments: three from the RV free wall and three from the RV septum. RV free wall longitudinal strain was averaged from the three free wall segments only.

Measurements for each subject included global and segmental myocardial strain parameters, including peak systolic strain, peak systolic strain rate, peak early diastolic strain rate, and their respective time-to-peak values. Global longitudinal, circumferential, and radial strain parameters were calculated by averaging the peak strain values.

In addition to full-thickness strain analysis, we performed layer-specific strain analysis in 20 randomly selected patients within the cohort. Three layer-specific 


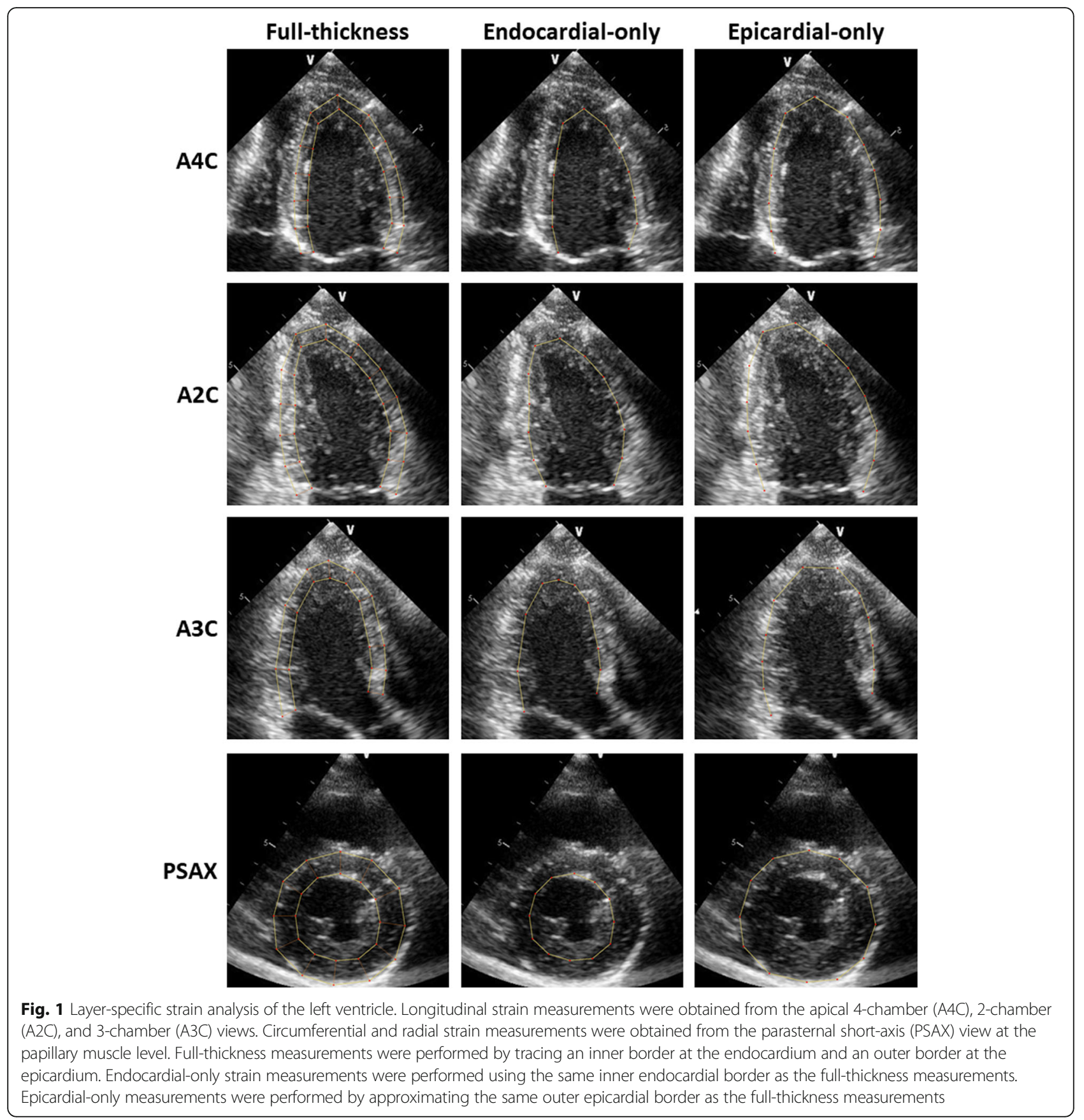

measurements were performed per subject: full-thickness, endocardial-only, and epicardial-only (Figures 1 and 2). The endocardial tracking boundary remained the same for the full-thickness and endocardial-only measurements. Analysis was performed in the same cardiac cycle for each of the layer-specific measurements.

\section{Reproducibility}

The global full-thickness strain measurements of the 20 patients who underwent layer-specific strain analyses were repeated by the same investigator (H.X.) several months after the initial analysis to determine intraobserver variability and performed by a second investigator (Z.Y.) to assess inter-observer variability.

\section{Statistical analysis}

Data were summarized as mean \pm standard deviation. Deformation parameters were compared between the different myocardial layers using one-way repeated measures analysis of variance (ANOVA). A $P$ value $<0.05$ was considered statistically significant. When the $P$ value was statistically significant, the paired $t$ test was used to 


\section{Longitudinal strain}
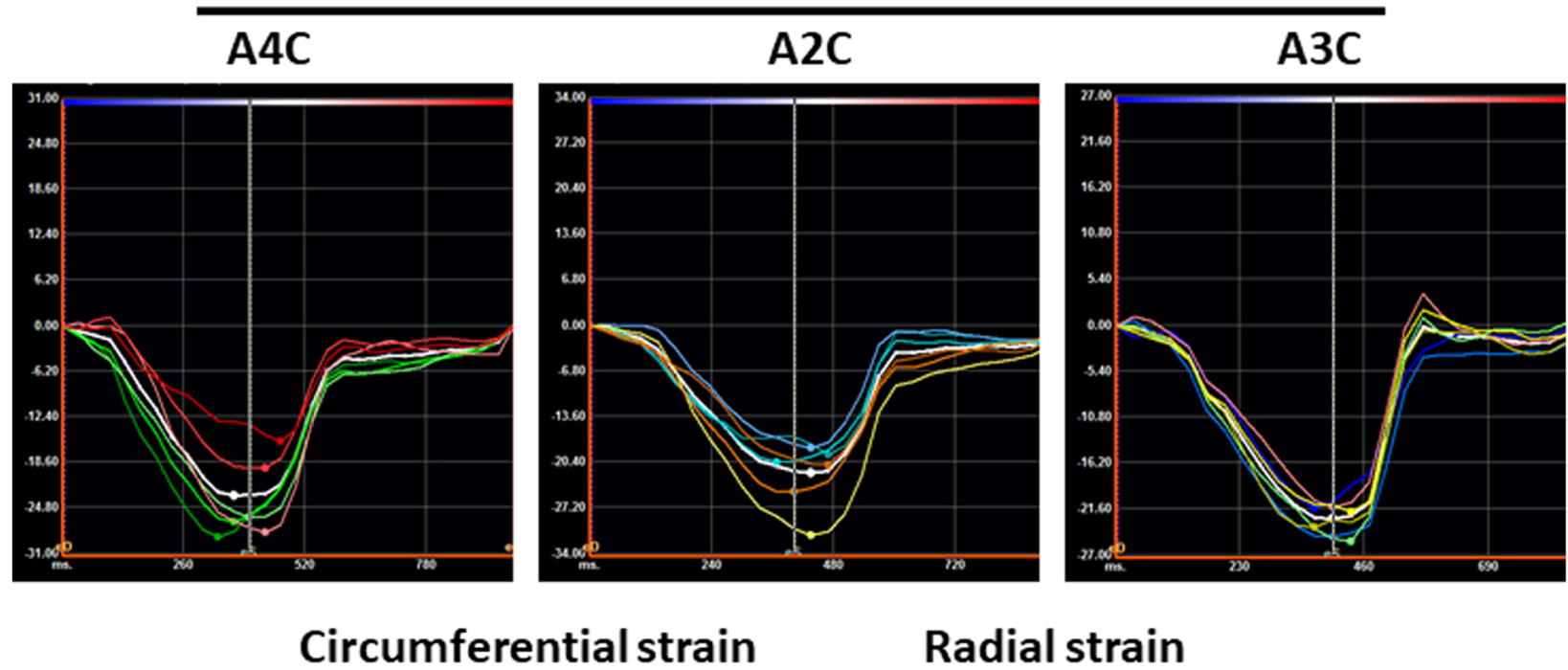

Radial strain
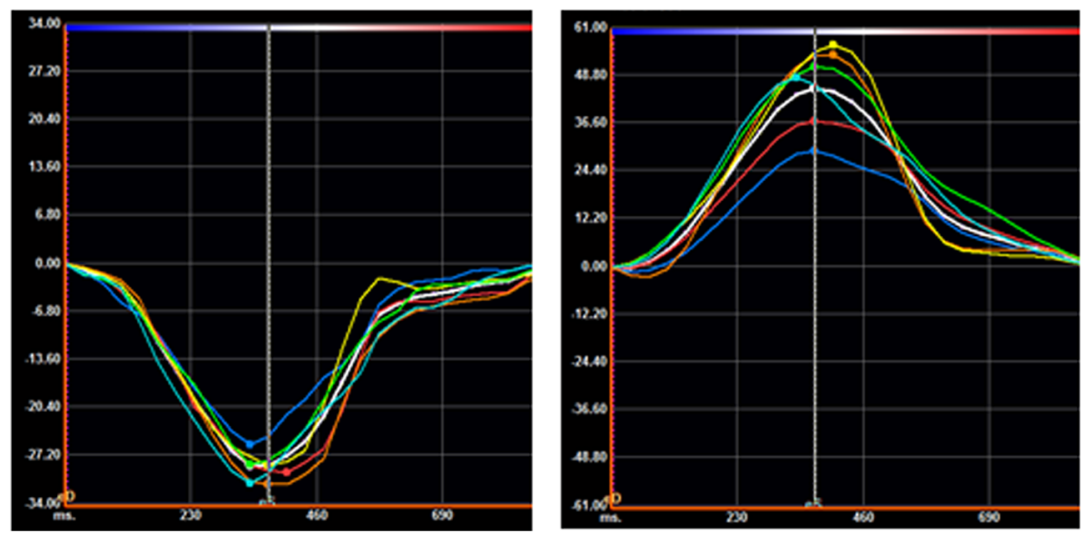

Fig. 2 Left ventricular strain curves. Average and segmental longitudinal strain curves were obtained from the apical 4-chamber (A4C), 2-chamber (A2C), and 3chamber (A3C) views. Average and segmental circumferential and radial strain curves were obtained from the parasternal long-axis view at the level of the papillary muscle. Global longitudinal and circumferential strain measurements were derived from the nadir of the average longitudinal and circumferential strain curves respectively. Global radial strain measurements were derived from the peak of the average radial strain curve

identify the pairwise difference. Differences between subgroups based on age were assessed with the TukeyKramer honestly significant difference test and between subgroups based on sex with the Student $t$ test. ShapiroWilks test was chosen for testing the normality of the data. Intra-class correlation coefficients (ICCs) with 95\% confidence intervals (CIs) were used to evaluate intraobserver and inter-observer variability. All statistical analyses were performed with JMP version 10.0 software (SAS Institute Inc., Cary, North Carolina).

This study was approved by the Mayo Clinic Institutional Review Board.

\section{Results}

Baseline characteristics

Baseline characteristics are presented in Table 1. Of the 106 patients screened, four patients were excluded due to suboptimal image quality. Full-thickness strain analysis was performed in the remaining 102 patients in the cohort of whom $63(62 \%)$ were female with a mean age of $39 \pm 15$ years. Among the 20 patients who underwent further layer-specific strain analysis, 10 (50\%) were female with a median age of $36 \pm 13$ years.

\section{Left ventricular full-thickness strain}

Table 2 displays the mean full-thickness LV global longitudinal, circumferential, and radial strain, systolic strain rate, early diastolic strain rate, and respective time-topeak measurements for patients overall and when stratified by age and sex. Mean LV global longitudinal, circumferential, and radial strain measured $-18.4 \pm 2.0 \%$, $-22.1 \pm 4.1 \%$, and $43.9 \pm 12.1 \%$, respectively. Women displayed higher mean LV longitudinal and circumferential systolic strain and longer mean LV longitudinal, 
Table 1 Baseline characteristics $(n=102)$

\begin{tabular}{ll}
\hline Characteristics & Full-Thickness Analysis \\
\hline Clinical & \\
Age (years) & $39 \pm 15$ \\
Female & $63(62 \%)$ \\
Body mass index $\left(\mathrm{kg} / \mathrm{m}^{2}\right)$ & $25.8 \pm 5.0$ \\
Heart rate $(\mathrm{bpm})$ & $73 \pm 14$ \\
Systolic blood pressure $(\mathrm{mmHg})$ & $115 \pm 15$ \\
Diastolic blood pressure $(\mathrm{mmHg})$ & $71 \pm 10$ \\
Echocardiographic & \\
LV end-diastolic diameter $(\mathrm{mm})$ & $47 \pm 4$ \\
LV end-systolic diameter $(\mathrm{mm})$ & $30 \pm 3$ \\
LV ejection fraction $(\%)$ & $64 \pm 4$ \\
E velocity (m/s) & $0.8 \pm 0.2$ \\
A velocity (m/s) & $0.5 \pm 0.1$ \\
Septal e' velocity $(\mathrm{m} / \mathrm{s})$ & $0.11 \pm 0.03$ \\
E/A ratio & $1.6 \pm 0.6$ \\
Septal E/e' ratio & $7.6 \pm 1.9$ \\
RV systolic pressure $(\mathrm{mmHg})$ & $25 \pm 4$ \\
\hline
\end{tabular}

Abbreviations: $A$ Late mitral inflow velocity, $E$ Early mitral inflow velocity, $e^{\prime}$ Mitral annulus tissue velocity, $L V$ Left ventricular, $R V$ Right ventricular The proportion of female subjects is expressed as number (\%). Continuous data are expressed as mean \pm standard deviation

circumferential, and radial time-to-peak strain compared with men. There was no consistent association between age and the various strain measurements, with LV longitudinal early diastolic strain rate being the only parameter that showed a statistically significant decrease with increasing age.

\section{Right ventricular strain}

Mean RV global and free wall longitudinal systolic strain, systolic strain rate, and early diastolic strain rate are outlined in Table 3. Mean RV global and free wall longitudinal systolic strain measured $-24.2 \pm 3.9 \%$ and $26.1 \pm 5.2 \%$, respectively. As with LV strain, mean RV global and free wall longitudinal systolic strain, systolic strain rate, and early diastolic strain rate were higher in women than in men and there was no consistent association with age.

\section{Left ventricular layer-specific strain}

Table 4 demonstrates mean LV global systolic strain, strain rate, and respective time-to-peak values depending on the layer analyzed for the 20 patients in whom layerspecific strain analysis was performed. Mean longitudinal, circumferential, and radial systolic strain, systolic strain rate, and early diastolic strain rate were lower in the epicardial-only layer compared with the fullthickness and endocardial-only layers $(P \leq 0.02)$. There
Table 2 Full-thickness left ventricular strain analysis

\begin{tabular}{|c|c|c|c|c|}
\hline Variable & $\begin{array}{l}\text { All subjects } \\
(n=102)\end{array}$ & $\begin{array}{l}\text { Men } \\
(n=39)\end{array}$ & Women $(n=63)$ & $P$ Value \\
\hline \multicolumn{5}{|l|}{ Longitudinal } \\
\hline S (\%) & $-18.4 \pm 2.0$ & $-17.4 \pm 1.5$ & $-19.0 \pm 2.0$ & $<0.001$ \\
\hline SRs (1/s) & $-0.99 \pm 0.12$ & $-0.95 \pm 0.09$ & $-1.02 \pm 0.12$ & 0.004 \\
\hline SRe $(1 / s)$ & $1.06 \pm 0.20$ & $0.99 \pm 0.15$ & $1.10 \pm 0.21$ & 0.008 \\
\hline S-TP (ms) & $378.7 \pm 43.4$ & $367.7 \pm 40.9$ & $385.7 \pm 43.8$ & 0.04 \\
\hline SRs-TP (ms) & $196.3 \pm 30.7$ & $189.9 \pm 33.5$ & $200.4 \pm 28.2$ & 0.09 \\
\hline SRe-TP (ms) & $502.9 \pm 50.5$ & $492.6 \pm 48.8$ & $509.4 \pm 50.8$ & 0.11 \\
\hline \multicolumn{5}{|l|}{ Circumferential } \\
\hline S (\%) & $-22.1 \pm 4.1$ & $-20.5 \pm 3.9$ & $-23.1 \pm 3.9$ & 0.002 \\
\hline SRs (1/s) & $-1.40 \pm 0.29$ & $-1.37 \pm 0.29$ & $-1.42 \pm 0.30$ & 0.35 \\
\hline SRe (1/s) & $1.39 \pm 0.35$ & $1.26 \pm 0.33$ & $1.47 \pm 0.34$ & 0.003 \\
\hline S-TP (ms) & $374.4 \pm 47.4$ & $360.9 \pm 43.0$ & $383.0 \pm 48.3$ & 0.02 \\
\hline SRs-TP (ms) & $217.0 \pm 38.4$ & $210.2 \pm 34.0$ & $221.4 \pm 40.6$ & 0.16 \\
\hline SRe-TP (ms) & $498.8 \pm 54.4$ & $491.1 \pm 47.8$ & $503.7 \pm 58.1$ & 0.26 \\
\hline \multicolumn{5}{|l|}{ Radial } \\
\hline S (\%) & $43.9 \pm 12.1$ & $43.7 \pm 11.5$ & $44.0 \pm 12.6$ & 0.89 \\
\hline SRs (1/s) & $2.17 \pm 0.62$ & $2.17 \pm 0.64$ & $2.17 \pm 0.62$ & 0.98 \\
\hline $\operatorname{SRe}(1 / \mathrm{s})$ & $-2.07 \pm 0.73$ & $-1.91 \pm 0.65$ & $-2.17 \pm 0.76$ & 0.09 \\
\hline S-TP (ms) & $381.1 \pm 66.1$ & $364.3 \pm 62.7$ & $391.9 \pm 66.5$ & 0.04 \\
\hline SRs-TP (ms) & $204.1 \pm 41.7$ & $192.4 \pm 33.7$ & $211.6 \pm 44.7$ & 0.02 \\
\hline SRe-TP (ms) & $507.2 \pm 62.9$ & $502.3 \pm 61.7$ & $510.3 \pm 64.0$ & 0.54 \\
\hline
\end{tabular}

Abbreviations: S Systolic strain, SRe Early diastolic strain rate, S-TP Time-to-peak strain, SRe-TP Time-to-peak early diastolic strain rate, SRs Systolic strain rate, SRs-TP Time-to-peak systolic strain rate

Continuous data are expressed as mean \pm standard deviation

Table 3 Full-thickness right ventricular strain analysis

\begin{tabular}{lllll}
\hline Variable & $\begin{array}{l}\text { All subjects } \\
(\mathrm{n}=102)\end{array}$ & $\begin{array}{l}\text { Men } \\
(\mathrm{n}=39)\end{array}$ & Women $(\mathrm{n}=63)$ & P Value \\
\hline Longitudinal & $(\mathrm{global})$ & & \\
S (\%) & $-24.2 \pm 3.9$ & $-22.3 \pm 3.4$ & $-25.4 \pm 3.8$ & 0.001 \\
SRs (1/s) & $-1.4 \pm 0.3$ & $-1.3 \pm 0.3$ & $-1.5 \pm 0.3$ & 0.01 \\
SRe (1/s) & $1.5 \pm 0.4$ & $1.3 \pm 0.3$ & $1.6 \pm 0.5$ & 0.001 \\
S-TP (ms) & $389.8 \pm 53.0$ & $379.5 \pm 54.3$ & $396.4 \pm 51.5$ & 0.12 \\
SRs-TP (ms) & $213.7 \pm 41.4$ & $205.7 \pm 38.4$ & $218.8 \pm 42.7$ & 0.13 \\
SRe-TP (ms) & $510.4 \pm 58.7$ & $499.7 \pm 53.5$ & $517.2 \pm 61.2$ & 0.15 \\
Longitudinal (free wall) & & & \\
S (\%) & $-26.1 \pm 5.2$ & $-24.5 \pm 4.4$ & $-27.1 \pm 5.4$ & 0.01 \\
SRs (1/s) & $-1.6 \pm 0.4$ & $-1.5 \pm 0.3$ & $-1.6 \pm 0.5$ & 0.07 \\
SRe (1/s) & $1.9 \pm 0.8$ & $1.6 \pm 0.8$ & $2.0 \pm 0.8$ & 0.01 \\
S-TP (ms) & $395.5 \pm 78.2$ & $384.7 \pm 64.6$ & $404.4 \pm 72.8$ & 0.25 \\
SRs-TP (ms) & $229.5 \pm 61.3$ & $224.6 \pm 60.3$ & $232.7 \pm 62.2$ & 0.52 \\
SRe-TP (ms) & $510.32 \pm 75.9$ & $502.1 \pm 80.7$ & $515.6 \pm 72.8$ & 0.41 \\
\hline
\end{tabular}

Abbreviations: $S$ Systolic strain, SRe Early diastolic strain rate, S-TP Time-to-peak strain, SRe-TP Time-to-peak early diastolic strain rate, SRs Systolic strain rate, SRs-TP Time-to-peak systolic strain rate

Continuous data are expressed as mean \pm standard deviation 
Table 4 Layer-specific global left ventricular strain analysis $(n=20)$

\begin{tabular}{|c|c|c|c|c|c|}
\hline Variable & Full-thickness & Endocardial-only & Epicardial-only & $P$ Value & Intra-class correlation coefficient ( $95 \%$ confidence interval) \\
\hline \multicolumn{6}{|l|}{ Longitudinal } \\
\hline S (\%) & $-19.2 \pm 2.2$ & $-19.1 \pm 2.1$ & $-16.5 \pm 1.6^{\mathrm{a}}$ & $<0.001$ & $0.97(0.71-0.94)$ \\
\hline SRs $(1 / \mathrm{s})$ & $-1.0 \pm 0.1$ & $-1.1 \pm 0.1$ & $-0.9 \pm 0.1^{\mathrm{a}}$ & $<0.001$ & $0.97(0.76-0.95)$ \\
\hline SRe (1/s) & $1.1 \pm 0.3$ & $1.1 \pm 0.3$ & $0.9 \pm 0.2^{a}$ & $<0.001$ & $0.97(0.85-0.97)$ \\
\hline S-TP (ms) & $380.1 \pm 42.6$ & $381.8 \pm 43.4$ & $373.5 \pm 41.5$ & 0.22 & $0.97(0.84-0.99)$ \\
\hline SRs-TP (ms) & $197.5 \pm 29.3$ & $201.4 \pm 32.6$ & $194.7 \pm 32.0$ & 0.59 & $0.91(0.83-0.96)$ \\
\hline SRe-TP (ms) & $499.4 \pm 46.6$ & $500.2 \pm 45.5$ & $496.9 \pm 47.7$ & 0.86 & $0.98(0.96-0.99)$ \\
\hline \multicolumn{6}{|l|}{ Circumferential } \\
\hline S (\%) & $-23.6 \pm 3.9$ & $-23.7 \pm 3.9$ & $-16.7 \pm 4.2^{a}$ & $<0.001$ & $0.96(0.50-0.89)$ \\
\hline SRs (1/S) & $-1.5 \pm 0.3$ & $-1.5 \pm 0.3$ & $-1.1 \pm 0.3^{\mathrm{a}}$ & $<0.001$ & $0.96(0.71-0.94)$ \\
\hline SRe $(1 / s)$ & $1.5 \pm 0.3$ & $1.4 \pm 0.3$ & $1.0 \pm 0.3^{\mathrm{a}}$ & $<0.001$ & $0.94(0.49-0.89)$ \\
\hline S-TP (ms) & $367.3 \pm 40.4$ & $365.8 \pm 41.6$ & $381.5 \pm 44.5$ & 0.44 & $0.71(0.43-0.87)$ \\
\hline SRs-TP (ms) & $223.4 \pm 32.2$ & $220.6 \pm 32.4$ & $219.7 \pm 43.3$ & 0.97 & $0.74(0.52-0.89)$ \\
\hline SRe-TP (ms) & $486.2 \pm 41.1$ & $483.0 \pm 40.0$ & $497.3 \pm 55.0$ & 0.57 & $0.73(0.48-0.88)$ \\
\hline \multicolumn{6}{|l|}{ Radial } \\
\hline S (\%) & $39.8 \pm 10.8$ & $50.9 \pm 22.9^{b}$ & $33.9 \pm 11.1^{c}$ & $<0.001$ & $0.69(0.18-0.82)$ \\
\hline SRs $(1 / s)$ & $1.8 \pm 0.5$ & $2.2 \pm 0.7^{b}$ & $1.9 \pm 0.7$ & 0.02 & $0.71(0.40-0.90)$ \\
\hline SRe (1/s) & $-1.7 \pm 0.7$ & $-2.4 \pm 1.5^{b}$ & $-1.6 \pm 0.8$ & 0.01 & $0.60(0.08-0.80)$ \\
\hline S-TP (ms) & $382.6 \pm 46.9$ & $414.0 \pm 70.6$ & $408.7 \pm 58.5$ & 0.18 & $0.66(0.30-0.84)$ \\
\hline SRs-TP (ms) & $212.1 \pm 53.0$ & $201.4 \pm 62.7$ & $202.3 \pm 47.0$ & 0.85 & $0.52(0.19-0.82)$ \\
\hline SRe-TP (ms) & $501.1 \pm 47.6$ & $491.3 \pm 54.7$ & $510.8 \pm 58.1$ & 0.34 & $0.81(0.63-0.92)$ \\
\hline
\end{tabular}

Abbreviations: S Systolic strain, SRe Early diastolic strain rate, S-TP Time-to-peak strain, SRe-TP Time-to-peak early diastolic strain rate, SRs Systolic strain rate, SRs-TP Time-to-peak systolic strain rate

Continuous data are expressed as mean \pm standard deviation

a $P<0.001$ when compared with full-thickness and endocardial-only groups

${ }^{b} P<0.05$ when compared with full-thickness group

${ }^{c} P<0.01$ when compared with full-thickness and endocardial-only groups

was no significant difference in strain measurements between the full-thickness and endocardial-only layers.

\section{Left ventricular segmental strain}

Mean segmental LV longitudinal and circumferential strain parameters corresponding to the different layers are presented in Table 5. Similar to the global strain analysis, segmental longitudinal and circumferential strain measurements were lowest in the epicardium compared with the full-thickness and endocardial-only layers $(P<0.001)$, which showed no significant difference between them $(P>0.05)$. In contrast, the segmental radial strain parameters demonstrated poor intra- and inter-observer variability based on the suboptimal intraclass correlation coefficients and are therefore not presented in Table 5 but available for review in Supplementary Table 2 .

\section{Left ventricular strain by ventricular level}

Longitudinal systolic strain and systolic strain rate were highest in the apex compared with the mid and basal LV in the endocardial-only and full-thickness layers $(P<$
0.001) but not in the epicardial-only layer (Table 6). There was no statistically significant difference in layerspecific longitudinal systolic strain and systolic strain rate between the mid and basal $\mathrm{LV}$.

\section{Reproducibility analysis}

The intra-observer variability and inter-observer variability for the various global full-thickness strain measurements are presented in Table 7 . Intra-class correlation coefficients were the highest for global longitudinal strain measurements, followed closely by circumferential strain measurements, and the lowest for global radial strain measurements.

\section{Discussion}

We have presented an analysis of ventricular strain performed within a real world cohort of patients with no structural heart disease using a vendor-independent speckle-tracking software. We measured longitudinal, circumferential, and radial systolic strain, strain rate, and respective time-to-peak values both globally and based on several parameters including age, sex, wall segment, 
Table 5 Layer-specific segmental left ventricular longitudinal and circumferential strain analysis $(n=20)$

\begin{tabular}{|c|c|c|c|c|c|}
\hline Variable & Full-thickness & Endocardial-only & Epicardial-only & $P$ Value & Intra-class correlation coefficient( $95 \%$ confidence interval \\
\hline \multicolumn{6}{|c|}{ Segmental longitudinal S (\%) } \\
\hline Anterior & $-19.8 \pm 3.1$ & $-19.6 \pm 3.1$ & $-17.1 \pm 2.8^{\mathrm{a}}$ & $<0.001$ & $0.96(0.82-0.96)$ \\
\hline Anteroseptal & $-19.1 \pm 3.1$ & $-19.1 \pm 2.9$ & $-16.5 \pm 2.4^{\mathrm{a}}$ & $<0.001$ & $0.90(0.68-0.93)$ \\
\hline Inferior & $-19.8 \pm 3.2$ & $-19.7 \pm 3.3$ & $-17.0 \pm 3.0^{\mathrm{a}}$ & $<0.001$ & $0.94(0.78-0.95)$ \\
\hline Lateral & $-19.8 \pm 3.0$ & $-19.6 \pm 2.9$ & $-15.9 \pm 3.2^{\mathrm{a}}$ & $<0.001$ & $0.94(0.65-0.92)$ \\
\hline Posterior & $-18.2 \pm 3.1$ & $-18.2 \pm 3.2$ & $-15.0 \pm 1.9^{\mathrm{a}}$ & $<0.001$ & $0.94(0.70-0.93)$ \\
\hline Septal & $-19.1 \pm 2.6$ & $-19.0 \pm 2.3$ & $-16.2 \pm 2.5^{\mathrm{a}}$ & $<0.001$ & $0.89(0.58-0.91)$ \\
\hline \multicolumn{6}{|c|}{ Segmental longitudinal SRs (1/s) } \\
\hline Anterior & $-1.1 \pm 0.2$ & $-1.1 \pm 0.2$ & $-0.9 \pm 0.2^{\mathrm{a}}$ & $<0.001$ & $0.94(0.80-0.96)$ \\
\hline Anteroseptal & $-1.1 \pm 0.2$ & $-1.1 \pm 0.2$ & $-1.0 \pm 0.2^{\mathrm{a}}$ & $<0.001$ & $0.92(0.77-0.94)$ \\
\hline Inferior & $-1.1 \pm 0.2$ & $-1.1 \pm 0.2$ & $-0.9 \pm 0.2^{\mathrm{a}}$ & $<0.001$ & $0.91(0.74-0.94)$ \\
\hline Lateral & $-1.1 \pm 0.2$ & $-1.1 \pm 0.2$ & $-0.9 \pm 0.2^{\mathrm{a}}$ & $<0.001$ & $0.94(0.68-0.93)$ \\
\hline Posterior & $-1.1 \pm 0.2$ & $-1.1 \pm 0.2$ & $-0.9 \pm 0.2^{\mathrm{a}}$ & $<0.001$ & $0.93(0.67-0.93)$ \\
\hline Septal & $-1.1 \pm 0.2$ & $-1.1 \pm 0.1$ & $-0.9 \pm 0.2^{\mathrm{a}}$ & $<0.001$ & $0.84(0.56-0.90)$ \\
\hline \multicolumn{6}{|c|}{ Segmental circumferential S (\%) } \\
\hline Anterior & $-24.6 \pm 7.0$ & $-24.7 \pm 6.7$ & $-15.3 \pm 5.0^{\mathrm{a}}$ & $<0.001$ & $0.92(0.53-0.90)$ \\
\hline Anteroseptal & $-24.9 \pm 6.0$ & $-25.2 \pm 6.4$ & $-16.9 \pm 5.2^{\mathrm{a}}$ & $<0.001$ & $0.94(0.60-0.91)$ \\
\hline Inferior & $-23.7 \pm 5.9$ & $-23.7 \pm 6.4$ & $-18.8 \pm 6.8^{\mathrm{a}}$ & $<0.001$ & $0.90(0.73-0.94)$ \\
\hline Lateral & $-22.7 \pm 6.6$ & $-22.7 \pm 6.4$ & $-15.2 \pm 5.2^{\mathrm{a}}$ & $<0.001$ & $0.92(0.63-0.92)$ \\
\hline Posterior & $-23.9 \pm 7.4$ & $-23.5 \pm 7.5$ & $-16.4 \pm 4.9^{\mathrm{a}}$ & $<0.001$ & $0.92(0.66-0.93)$ \\
\hline Septal & $-22.0 \pm 5.8$ & $-22.3 \pm 5.7$ & $-17.7 \pm 6.5^{\mathrm{a}}$ & $<0.001$ & $0.84(0.59-0.91)$ \\
\hline \multicolumn{6}{|c|}{ Segmental circumferential SRs (1/s) } \\
\hline Anterior & $-1.5 \pm 0.5$ & $-1.5 \pm 0.5$ & $-1.0 \pm 0.4^{\mathrm{a}}$ & $<0.001$ & $0.86(0.54-0.90)$ \\
\hline Anteroseptal & $-1.6 \pm 0.5$ & $-1.6 \pm 0.5$ & $-1.1 \pm 0.5^{\mathrm{a}}$ & $<0.001$ & $0.95(0.75-0.94)$ \\
\hline Inferior & $-1.6 \pm 0.5$ & $-1.6 \pm 0.5$ & $-1.2 \pm 0.5^{\mathrm{a}}$ & $<0.001$ & $0.95(0.82-0.96)$ \\
\hline Lateral & $-1.4 \pm 0.4$ & $-1.4 \pm 0.4$ & $-1.0 \pm 0.4^{\mathrm{a}}$ & $<0.001$ & $0.83(0.50-0.89)$ \\
\hline Posterior, $1 / \mathrm{s}$ & $-1.5 \pm 0.6$ & $-1.5 \pm 0.6$ & $-1.1 \pm 0.5^{\mathrm{a}}$ & $<0.001$ & $0.96(0.82-0.96)$ \\
\hline Septal, 1/s & $-1.4 \pm 0.5$ & $-1.4 \pm 0.4$ & $-1.2 \pm 0.5^{b}$ & 0.009 & $0.86(0.70-0.93)$ \\
\hline
\end{tabular}

Abbreviations: S Systolic strain, SRs Systolic strain rate

Continuous data are expressed as mean \pm standard deviation

a $P<0.001$ when compared with full-thickness and endocardial-only groups

${ }^{\mathrm{b}} P<0.05$ when compared with full-thickness and endocardial-only groups

ventricular level, and myocardial layer, while providing information on the reproducibility of these measurements. Full-thickness mean LV global longitudinal, circumferential, and radial systolic strain measured $-18.4 \pm 2.0 \%$, $22.1 \pm 4.1 \%$, and $43.9 \pm 12.1 \%$ respectively, while mean RV global and free wall longitudinal systolic strain measured $-24.2 \pm 3.9 \%$ and $-26.1 \pm 5.2 \%$ respectively.

Our results were generally similar to those found in previous studies with some differences. In a meta-analysis of 2597 healthy subjects from 24 studies, average values of LV global longitudinal, circumferential, and radial strain overlapped with our findings and were reported to be $19.7 \%$ (95\% CI $-20.4 \%$ to $-18.9 \%),-23.3 \%$ (95\% CI $24.6 \%$ to $-22.1 \%$ ), and $47.3 \%$ (95\% CI $43.6 \%$ to $51.0 \%$ )
[21]. Differences in characteristics of patients included in the meta-analysis as well as differences in vendor software used for the strain analyses could account for the corresponding differences in results [27-31].

On the other hand, in a study of 549 healthy subjects enrolled in 22 European institutions in whom strain was measured using the same vendor-independent 2D Cardiac Performance Analysis software by TomTec, LV global longitudinal strain was similar at $-22.5 \pm 2.7 \%$ while absolute global circumferential and radial strain values were much higher at $-31.9 \pm 4.5$, and $37.4 \pm 8.4 \%$ respectively compared to our study [18]. This could be related to the differences in method used to measure circumferential and radial strain. While we analyzed 
Table 6 Layer-specific left ventricular longitudinal strain analysis by ventricular level $(n=20)$

\begin{tabular}{llllll}
\hline Variable & Full-thickness & Endocardial-only & Epicardial-only & $P$ Value & Intra-class correlation coefficient(95\% confidence interval) \\
\hline Level-specific longitudinal S (\%) & & & & \\
Basal & $-18.1 \pm 2.1$ & $-18.2 \pm 2.0$ & $-16.4 \pm 2.0^{\mathrm{a}}$ & $<0.001$ & $0.90(0.68-0.93)$ \\
Mid & $-18.2 \pm 2.4$ & $-18.1 \pm 2.4$ & $-16.7 \pm 2.1^{\mathrm{b}}$ & $<0.001$ & $0.96(0.86-0.97)$ \\
Apical & $-21.6 \pm 3.1^{\mathrm{c}}$ & $-21.3 \pm 3.2^{\mathrm{c}}$ & $-16.8 \pm 2.1^{\mathrm{b}}$ & $<0.001$ & $0.91(0.23-0.83)$ \\
$\begin{array}{l}P \text { value } \\
\text { Level-specific longitudinal SRs (1/s) }\end{array}$ & $<0.001$ & 0.09 & & \\
Basal & $-1.01 \pm 0.16$ & $-1.03 \pm 0.15$ & $-0.97 \pm 0.16^{\mathrm{a}}$ & 0.06 & $0.92(0.82-0.96)$ \\
Mid & $-0.99 \pm 0.14$ & $-1.00 \pm 0.14$ & $-0.91 \pm 0.11^{\mathrm{a}}$ & $<0.001$ & $0.96(0.86-0.97)$ \\
Apical & $-1.21 \pm 0.26^{\mathrm{c}}$ & $-1.20 \pm 0.16^{\mathrm{c}}$ & $-0.95 \pm 0.16^{\mathrm{b}}$ & $<0.001$ & $0.87(-0.11-0.75)$ \\
$P$ value & $<0.001$ & $<0.001$ & 0.07 & &
\end{tabular}

Abbreviations: S Systolic strain, SRs Systolic strain rate

Continuous data are expressed as mean \pm standard deviation

${ }^{a} P<0.005$ when compared with full-thickness and endocardial-only

${ }^{\mathrm{b}} P<0.001$ when compared with full-thickness and endocardial-only groups

c $P<0.001$ when compared with basal and mid left ventricular levels

strain at the level of the papillary muscle, that study also included the basal and apical levels for analysis as well.

The results of our analysis confirm a number of characteristics of ventricular systolic strain that have been shown previously in separate studies but have infrequently been demonstrated within the same cohort, as we have done in this study. First, absolute values of the various ventricular strain parameters are higher in women than in men $[7,11,13,16-18]$ and in the RV compared with the LV [12]. Furthermore, systolic strain parameters are higher in the endocardium than in the epicardium and in apex compared with the base when measured in the endocardial layer $[7,9,10,14,16,17]$.

Table $\mathbf{7}$ Intra- and inter-observer variability of global fullthickness left ventricular strain

\begin{tabular}{|c|c|c|}
\hline \multirow[b]{2}{*}{ Variable } & \multicolumn{2}{|c|}{ Intra-class correlation coefficient( $95 \%$ confidence interval) } \\
\hline & Intra-observer variability & Inter-observer variability \\
\hline \multicolumn{3}{|c|}{ Longitudinal } \\
\hline S & $0.998(0.994-0.999)$ & $0.996(0.989-0.998)$ \\
\hline SRs & $0.995(0.988-0.998)$ & $0.982(0.956-0.993)$ \\
\hline SRe & $0.964(0.913-0.986)$ & $0.994(0.984-0.997)$ \\
\hline \multicolumn{3}{|c|}{ Circumferential } \\
\hline S & $0.997(0.994-0.999)$ & $0.993(0.983-0.997)$ \\
\hline SRs & $0.994(0.984-0.997)$ & $0.975(0.940-0.990)$ \\
\hline SRe & $0.967(0.919-0.987)$ & $0.967(0.919-0.987)$ \\
\hline \multicolumn{3}{|l|}{ Radial } \\
\hline S & $0.857(0.751-0.933)$ & $0.845(0.727-0.927)$ \\
\hline SRs & $0.906(0.782-0.961)$ & $0.898(0.818-0.953)$ \\
\hline SRe & $0.808(0.668-0.909)$ & $0.871(0.477-0.918)$ \\
\hline
\end{tabular}

Abbreviations: S Systolic strain, SRe Early diastolic strain rate, SRs Systolic strain rate
Compared to the base, the apex is smaller and is subject to less wall stress, which may result in relatively higher global longitudinal strain [9]. In addition, global longitudinal systolic strain is the most reproducible measure of strain, followed closely by global circumferential systolic strain, whereas global radial systolic strain demonstrates suboptimal intra- and inter-observer variability [12]. Radial strain may be less reproducible than longitudinal strain since the spread of myocardium is smaller in this direction resulting in fewer speckles to track and therefore making them more vulnerable to the impact of uncertain boundaries. Furthermore, unlike longitudinal strain, circumferential and radial strain represents myocardial fiber displacement that is not occurring parallel to the ultrasound beam [32].

The impact of age on strain measurements is less clear. In our study, there was no statistically significant difference in full-thickness LV or RV systolic strain or systolic strain rate between the three age categories examined. Statistical significance was only found in the LV early diastolic strain rate and RV time-to-peak systolic strain rate, which given the isolated nature of these findings, have unclear clinical significance. These findings may be due to our small sample size. Nevertheless, prior studies have also demonstrated conflicting results regarding the influence of age on strain, with one study showing no significant correlation [33] while several other studies suggested a decline in LV or RV longitudinal or radial systolic strain and systolic strain rate with age $[11,12,34]$.

More recent studies suggest that there may be a more complex interaction between age and strain, depending on the type of strain as well as the level and layer within the ventricle [7, 17, 18]. Longitudinal systolic strain appears to generally decrease with age whereas 
circumferential and radial systolic strain measurements appear to increase $[17,18]$. However, longitudinal systolic strain does not appear to uniformly decrease within the ventricle and instead decreases more in the base and increases more in the apex with age [7, 17]. Regional variability in strain and differences in the mean age of subjects in a given cohort could partly account for differences in age-related changes in strain observed between studies. Of note, in the meta-analysis of 24 studies that reported normal ranges of LV systolic strain, only two included subjects with a mean age of $\geq 65$ years [21]. Given the increasing proportion of patients aged $\geq 65$ years referred for echocardiography, more precise characterization of strain in this population would be a worthwhile area of further investigation.

Similarly, use of the vendor-independent 2D Cardiac Performance Analysis software (TomTec Imaging System, Munich, Germany) is under-represented in studies that report normal reference ranges for strain despite its increasing use in clinical practice and research. Among the 28 data sets included in the meta-analysis outlining normal ranges of LV strain, 23 of them used EchoPAC software (GE Healthcare, Milwaukee, WI) and none of the remaining 5 data sets used the 2D Cardiac Performance Analysis software for strain analysis [21]. To date, only one other study has presented normal ranges of LV strain using this software and these patients were healthy subjects referred to various institutions in Europe [18]. Our study not only increases the representation of this software in reporting reference ranges of LV strain but also provides measurements of systolic and early diastolic strain rates, respective time-to-peak values, layerspecific strain, and RV longitudinal global and free wall systolic strain in a North American cohort, which has not been described previously.

\section{Limitations}

The present study must be interpreted with the following limitations in mind. The sample size of our study was small. Strain analysis was performed retrospectively. Patients in our cohort had a clinically justified reason to be referred for echocardiography, which may introduce selection bias. We could not fully exclude patients with non-cardiac comorbidities that could have potentially influenced myocardial deformation in some way. Our images were acquired using the Vivid 7 GE ultrasound system and analyzed using TomTec Imaging Systems, which could limit the generalizability of the findings. Furthermore, our strain measurements could have been slightly different if another definition of end-diastole or end-systole were chosen. However, such differences would likely be negligible in our cohort of patients with no regional wall motion abnormalities. In addition, the speckle-tracking software used in this study follows an algorithm that relies predominantly on the inner boundary traced even when both an inner and outer boundary are provided, which explains the similar strain measurements between the full-thickness and endocardial-only layers. As well, for our analysis of layer-specific strain, the mid-myocardial layer was not assessed individually as it was difficult to isolate the mid-myocardial layer without significant overlap with the endocardial and epicardial layers. Nevertheless, a prior study demonstrated good agreement in global longitudinal strain by default vendor layer in the mid myocardium for the GE platform and the endocardium for TomTec [35]. Finally, concomitant measurements of ventricular strain using other modalities such as magnetic resonance imaging or sonomicrometry were not available for comparison as this was beyond the scope of our study. Instead, we aimed to demonstrate the expected range of ventricular strain measured by 2D speckle-tracking echocardiography in patients without structural heart disease.

\section{Conclusions}

We performed an analysis of ventricular strain in a cohort of patients with no evidence of structural heart disease using the vendor-independent 2D Cardiac Performance Analysis software by TomTec. Women demonstrate higher absolute values of strain than men. Longitudinal strain is the most reproducible measure of strain while radial strain is the least reproducible. Strain is highest at the apex and in the endocardium. Further study is warranted to clarify the role of age on strain, particularly in elderly individuals who represent the majority of patients referred for cardiovascular evaluation. Our study provides reference ranges for various parameters of strain that may be encountered in a real world cohort of patients referred for echocardiography.

\section{Supplementary information}

Supplementary information accompanies this paper at https://doi.org/10. 1186/s12872-020-01559-1.

Additional file 1: Supplementary Table 1. Indications for echocardiography

Additional file 2: Supplementary Table 2. Layer-specific left ventricular segmental radial strain analysis $(n=20)$

\section{Abbreviations}

2D: 2-dimensional; A: Late mitral inflow velocity; E: Early mitral inflow velocity; e': Mitral annulus tissue velocity; EF: Ejection fraction; ICC: Intra-class correlation coefficient; LV: Left ventricle; RV: Right ventricle; S: Systolic strain; S-TP: Time-to-peak strain; SRe: Early diastolic strain rate; SRe-TP: Time-to-peak early diastolic strain rate; SRs: Systolic strain rate; SRs-TP: Systolic strain rate

\section{Acknowledgements}

Not applicable.

Authors' contributions

HX selected the patients, performed the speckle-tracking analysis and drafted the manuscript. PAP, CDS, and JJT interpreted the data and revised the 
manuscript. SSC performed the statistical analysis. ZY performed the interobserver variability analysis. DFY revised the manuscript. HRV designed the study, analyzed the data and revised the manuscript. The authors have read and approved the manuscript.

\section{Funding}

Not applicable.

\section{Availability of data and materials}

Public access to the database is closed. All requests for raw and analyzed data and related materials will be reviewed by the Mayo Clinic legal department to verify whether the request is subject to any intellectual property or confidentiality obligations. Requests for patient-related data not included in the paper will not be considered. Any data and materials that can be shared will be released via a Material Transfer Agreement.

\section{Ethics approval and consent to participate}

The Mayo Clinic Institutional Review Board approved the study and waived the requirement to obtain informed consent in accordance with 45 CFR 46.116 and Health Insurance Portability and Accountability Act (HIPAA) regulations.

\section{Consent for publication}

Not applicable.

\section{Competing interests}

The authors declare no competing interests.

\section{Author details}

'Department of Ultrasound, Xinqiao Hospital, Third Military Medical University, Chongqing, China. ${ }^{2}$ Department of Cardiovascular Diseases, Mayo Clinic, Rochester, Minnesota, 200 First St SW, Rochester, MN 55905, USA. ${ }^{3}$ Hypertension Unit, Department of Medical Sciences, University of Torino, Torino, Italy. ${ }^{4}$ Division of Biomedical Statistics and Informatics, Mayo Clinic, Rochester, MN, USA.

\section{Received: 26 December 2019 Accepted: 28 May 2020} Published online: 05 June 2020

\section{References}

1. Haugaa KH, Grenne BL, Eek CH, Ersboll M, Valeur N, Svendsen JH, et al. Strain echocardiography improves risk prediction of ventricular arrhythmias after myocardial infarction. JACC Cardiovasc Imaging. 2013;6:841-50.

2. Kalam K, Otahal P, Marwick TH. Prognostic implications of global LV dysfunction: a systematic review and meta-analysis of global longitudinal strain and ejection fraction. Heart. 2014;100:1673-80.

3. Marwick TH, Shah SJ, Thomas JD. Myocardial strain in the assessment of patients with heart failure: a review. JAMA Cardiol. 2019;4:287-94.

4. Russo C, Jin Z, Elkind MS, Rundek T, Homma S, Sacco RL, et al. Prevalence and prognostic value of subclinical left ventricular systolic dysfunction by global longitudinal strain in a community-based cohort. Eur J Heart Fail. 2014;16:1301-9.

5. Stanton T, Leano R, Marwick TH. Prediction of all-cause mortality from global longitudinal speckle strain: comparison with ejection fraction and wall motion scoring. Circ Cardiovasc Imaging. 2009;2:356-64.

6. Zhang KW, French B, May Khan A, Plappert T, Fang JC, Sweitzer NK, et al. Strain improves risk prediction beyond ejection fraction in chronic systolic heart failure. J Am Heart Assoc. 2014;3:e000550.

7. Abou R, Leung M, Khidir MJH, Wolterbeek R, Schalij MJ, Ajmone Marsan N, et al. Influence of aging on level and layer-specific left ventricular longitudinal strain in subjects without structural heart disease. Am J Cardiol. 2017;120:2065-72.

8. Adamu U, Schmitz F, Becker M, Kelm M, Hoffmann R. Advanced speckle tracking echocardiography allowing a three-myocardial layer-specific analysis of deformation parameters. Eur J Echocardiogr. 2009;10:303-8.

9. Bogaert J, Rademakers FE. Regional nonuniformity of normal adult human left ventricle. Am J Physiol Heart Circ Physiol. 2001;280:H610-20.

10. Carasso S, Biaggi P, Rakowski H, Mutlak D, Lessick J, Aronson D, et al. Velocity vector imaging: standard tissue-tracking results acquired in normals--the WI-STRAIN study. J Am Soc Echocardiogr. 2012;25:543-52.
11. Dalen $H$, Thorstensen $A$, Aase $S A$, Ingul CB, Torp $H$, Vatten $L J$, et al. Segmental and global longitudinal strain and strain rate based on echocardiography of 1266 healthy individuals: the HUNT study in Norway. Eur J Echocardiogr. 2010;11:176-83.

12. Fine NM, Shah AA, Han IY, Yu Y, Hsiao JF, Koshino Y, et al. Left and right ventricular strain and strain rate measurement in normal adults using velocity vector imaging: an assessment of reference values and intersystem agreement. Int J Cardiovasc Imaging. 2013;29:571-80.

13. Hurlburt HM, Aurigemma GP, Hill JC, Narayanan A, Gaasch WH, Vinch CS, et al. Direct ultrasound measurement of longitudinal, circumferential, and radial strain using 2-dimensional strain imaging in normal adults. Echocardiography. 2007;24:723-31.

14. Leitman M, Lysiansky M, Lysyansky P, Friedman Z, Tyomkin V, Fuchs T, et al. Circumferential and longitudinal strain in 3 myocardial layers in normal subjects and in patients with regional left ventricular dysfunction. J Am Soc Echocardiogr. 2010;23(1):64-70.

15. Moore CC, Lugo-Olivieri CH, McVeigh ER, Zerhouni EA. Three-dimensional systolic strain patterns in the normal human left ventricle: characterization with tagged MR imaging. Radiology. 2000;214:453-66.

16. Moreira HT, Nwabuo CC, Armstrong AC, Kishi S, Gjesdal O, Reis JP, et al. Reference ranges and regional patterns of left ventricular strain and strain rate using two-dimensional speckle-tracking echocardiography in a healthy middle-aged black and white population: the CARDIA study. J Am Soc Echocardiogr. 2017;30:647-58.e2.

17. Reckefuss N, Butz T, Horstkotte D, Faber L. Evaluation of longitudinal and radial left ventricular function by two-dimensional speckle-tracking echocardiography in a large cohort of normal probands. Int J Cardiovasc Imaging. 2011;27:515-26.

18. Sugimoto T, Dulgheru R, Bernard A, llardi F, Contu L, Addetia K, et al. Echocardiographic reference ranges for normal left ventricular 2D strain: results from the EACVI NORRE study. Eur Heart J Cardiovasc Imaging. 2017; 18:833-40.

19. Altiok E, Neizel M, Tiemann S, Krass V, Kuhr K, Becker M, et al. Quantitative analysis of endocardial and epicardial left ventricular myocardial deformation-comparison of strain-encoded cardiac magnetic resonance imaging with two-dimensional speckle-tracking echocardiography. J Am Soc Echocardiogr. 2012;25:1179-88.

20. Marwick TH, Leano RL, Brown J, Sun JP, Hoffmann R, Lysyansky P, et al. Myocardial strain measurement with 2-dimensional speckle-tracking echocardiography: definition of normal range. JACC Cardiovasc Imaging. 2009;2:80-4

21. Yingchoncharoen T, Agarwal S, Popovic ZB, Marwick TH. Normal ranges of left ventricular strain: a meta-analysis. J Am Soc Echocardiogr. 2013;26:185-91.

22. Lang RM, Badano LP, Mor-Avi V, Afilalo J, Armstrong A, Ernande L, et al. Recommendations for cardiac chamber quantification by echocardiography in adults: an update from the American Society of Echocardiography and the European Association of Cardiovascular Imaging. J Am Soc Echocardiogr. 2015;28:1-39.

23. Nagueh SF, Smiseth OA, Appleton CP, Byrd BF 3rd, Dokainish H, Edvardsen $T$, et al. Recommendations for the evaluation of left ventricular diastolic function by echocardiography: an update from the American Society of Echocardiography and the European Association of Cardiovascular Imaging. J Am Soc Echocardiogr. 2016;29:277-314.

24. Zoghbi WA, Adams D, Bonow RO, Enriquez-Sarano M, Foster E, Grayburn PA, et al. Recommendations for noninvasive evaluation of native Valvular regurgitation: a report from the American Society of Echocardiography developed in collaboration with the Society for Cardiovascular Magnetic Resonance. J Am Soc Echocardiogr. 2017;30:303-71.

25. Baumgartner H, Hung J, Bermejo J, Chambers JB, Evangelista A, Griffin BP, et al. Echocardiographic assessment of valve stenosis: EAE/ASE recommendations for clinical practice. J Am Soc Echocardiogr. 2009;22:1-23.

26. Rudski LG, Lai WW, Afilalo J, Hua L, Handschumacher MD, Chandrasekaran K et al. Guidelines for the echocardiographic assessment of the right heart in adults: a report from the American Society of Echocardiography endorsed by the European Association of Echocardiography, a registered branch of the European Society of Cardiology, and the Canadian Society of Echocardiography. J Am Soc Echocardiogr. 2010;23:685-713.

27. Farsalinos KE, Daraban AM, Unlu S, Thomas JD, Badano LP, Voigt JU. Headto-head comparison of global longitudinal strain measurements among nine different vendors: the EACVI/ASE inter-vendor comparison study. J Am Soc Echocardiogr. 2015;28:1171-81 e2. 
28. Gayat E, Ahmad H, Weinert L, Lang RM, Mor-Avi V. Reproducibility and intervendor variability of left ventricular deformation measurements by threedimensional speckle-tracking echocardiography. J Am Soc Echocardiogr. 2011:24:878-85.

29. Amzulescu MS, De Craene $M$, Langet $H$, et al. Myocardial strain imaging: review of general principles, validation, and sources of discrepancies. Eur Heart J Cardiovasc Imaging. 2019;20:605-19.

30. Mirea O, Pagourelias ED, Duchenne J, et al. Variability and reproducibility of segmental longitudinal strain measurement: a report from the EACVI-ASE strain standardization task force. JACC Cardiovasc Imaging. 2018;11:15-24.

31. D'hooge J, Barbosa D, Gao H, et al. Two-dimensional speckle tracking echocardiography: standardization efforts based on synthetic ultrasound data. Eur Heart J Cardiovasc Imaging. 2016;17:693-701.

32. Manovel A, Dawson D, Smith B, Nihoyannopoulos P. Assessment of left ventricular function by different speckle-tracking software. Eur 」 Echocardiogr. 2010;11:417-21.

33. Sun JP, Popovic ZB, Greenberg NL, Xu XF, Asher CR, Stewart WJ, et al. Noninvasive quantification of regional myocardial function using Dopplerderived velocity, displacement, strain rate, and strain in healthy volunteers: effects of aging. J Am Soc Echocardiogr. 2004;17:132-8.

34. Kuznetsova T, Herbots L, Richart T, D'Hooge J, Thijs L, Fagard RH, et al. Left ventricular strain and strain rate in a general population. Eur Heart J. 2008; 29:2014-23.

35. Ramlogan S, Aly D, France R, Schmidt S, Hinzman J, Sherman A, et al. Reproducibility and Intervendor agreement of left ventricular global systolic strain in children using a layer-specific analysis. J Am Soc Echocardiogr. 2020;33:110-9.

\section{Publisher's Note}

Springer Nature remains neutral with regard to jurisdictional claims in published maps and institutional affiliations.

Ready to submit your research? Choose BMC and benefit from:

- fast, convenient online submission

- thorough peer review by experienced researchers in your field

- rapid publication on acceptance

- support for research data, including large and complex data types

- gold Open Access which fosters wider collaboration and increased citations

- maximum visibility for your research: over $100 \mathrm{M}$ website views per year

At $\mathrm{BMC}$, research is always in progress.

Learn more biomedcentral.com/submissions 